\title{
Controle Químico de Plantas Daninhas Aquáticas: Alternanthera philoxeroides, Enhydra anagallis E Pycreus decumbens ${ }^{1}$
}

\author{
Chemical Control of Aquatic Weed Plants: Alternanthera philoxeroides, Enhydra anagallis \\ and Pycreus decumbens
}

\begin{abstract}
COSTA, N.V. ${ }^{2}$, CARDOSO, L.A. ${ }^{4}$, MARCHI, S.R. ${ }^{2}$, DOMINGOS, V.D. ${ }^{2}$ e MARTINS, D. ${ }^{3}$
RESUMO - As espécies de plantas aquáticas podem causar inúmeros inconvenientes ao uso múltiplo da água quando elas se desenvolvem desordenadamente. Assim, o objetivo deste trabalho foi avaliar a eficiência de diferentes herbicidas no controle químico de plantas de Alternanthera philoxeroides, Enhydra anagallis e Pycreus decumbens em caixa-d'água. Quando as plantas atingiram o seu pleno desenvolvimento (antes do florescimento), foram aplicados, nas espécies Alternanthera philoxeroides e Enhydra anagallis, os herbicidas: 2,4-D amina (U46 D FLUID 720) a 2.880 g e.a. ha-1 ${ }^{-1}$ diquat (REWARD 240) a 480 g i.a. ha ${ }^{-1}$; imazapyr (ARSENAL 250) a 500 e 750 g e.a. ha ${ }^{-1}$; glyphosate (RODEO 480) a 3.360 g e.a. ha ${ }^{-1}$ com e sem o surfatante Aterbane BR $\left(0,5 \% \mathrm{v} \mathrm{v}^{-1}\right)$; glyphosate + diquat $\left(3.360+480 \mathrm{~g}\right.$ i. $/$ e.a. ha $\left.{ }^{-1}\right)$; glyphosate $+2,4-\mathrm{D}$ $(3.360+2.880$ g e.a. ha-1 $)$; e diquat $+2,4-\mathrm{D}\left(480+2.880 \mathrm{~g}\right.$ i. $/$ e.a. ha $\left.{ }^{-1}\right)$, além de uma testemunha sem aplicação de herbicida. Para a espécie Pycreus decumbens foram aplicados: 2,4-D amina (U-46 D FLUID 720) a 2.880 g e.a. ha ${ }^{-1}$; diquat (REWARD 240) a 480 g i.a. ha ${ }^{-1}$; propanil (STAM 480) a 2.880 g i.a. ha-1 ${ }^{-1}$ glyphosate (RODEO 480) a 3.360 g e.a. ha ${ }^{-1}$ mais o surfatante Aterbane BR $\left(0,5 \% \mathrm{v} \mathrm{v}^{-1}\right)$; glyphosate + propanil $\left(3.360+2.880 \mathrm{~g}\right.$ i./e.a. ha $\left.{ }^{-1}\right)$; glyphosate + diquat $\left(3.360+480\right.$ g i./e.a. ha $\left.{ }^{-1}\right)$; glyphosate $+2,4-\mathrm{D}\left(3.360+2.880\right.$ g e.a. ha h $^{-1}$; propanil + 2,4-D $(2.880+2.880$ g i./e.a. ha-1); e diquat + 2,4-D $(480+2.880 \mathrm{~g}$ i./e.a. ha-1 $)$, além de uma testemunha sem aplicação de herbicida. Os herbicidas foram aplicados com um pulverizador estacionário, pressurizado a ar comprimido e equipado com um reservatório de 2 litros, pontas Teejet XR11002VS, com um consumo de calda de $200 \mathrm{~L} \mathrm{ha}^{-1}$. As avaliações de controle das plantas daninhas foram visuais, por meio de uma escala de percentual de notas, além de se avaliar a massa seca das plantas. Verificou-se que o controle químico apresenta-se como uma boa alternativa de manejo para as espécies A. philoxeroides, E. anagallis e $P$. decumbens, e a mistura de herbicidas pode aumentar a eficiência de controle. E. anagallis apresentou alta sensibilidade à ação dos herbicidas; entretanto, as espécies $A$. philoxeroides e $P$. decumbens evidenciaram alta capacidade de regeneração, principalmente quando se utilizaram herbicidas de ação de contato.
\end{abstract}

Palavras-chave: 2,4-D, diquat, imazapyr, glyphosate e planta daninha.

ABSTRACT - Aquatic plants species can cause countless inconveniences to the multiple use of water, when growing inordinately. The objective of this research was to evaluate the effect of different herbicides on Alternanthera philoxeroides, Enhydra anagallis and Pycreus decumbens plants under water tank conditions. When the plants reached full development (before flowering) the species Alternanthera philoxeroides and Enhydra anagallis were applied: 2,4-D amina (U-46 D FLUID 720) at 2,880 g a.e. ha ; diquat (REWARD 240) at $480 \mathrm{~g}$ a.i. $\mathrm{ha}^{-1}$; imazapyr (ARSENAL 250) at 500 and $750 \mathrm{~g}$ a.e. ha ${ }^{-1}$; glyphosate (RODEO 480) at 3,360 g a.e. ha (1 $^{-1}$ with and without the surfactant Aterbane BR (0.5\% v $\left.v^{-1}\right)$; glyphosate + diquat at $(3.360+$ $480 \mathrm{~g}$ a.i./e. $\left.\mathrm{ha}^{-1}\right)$; glyphosate $+2,4-\mathrm{D}$ at $\left(3,360+2,880 \mathrm{~g}\right.$ a.e. $\left.\mathrm{ha}^{-1}\right)$, diquat $+2,4-\mathrm{D}$ at $(480+$ 2,880 $\mathrm{g}$ a.i./ e. $\mathrm{ha}^{-1}$ ) and a control without herbicide. The following treatments were applied to

1 Recebido para publicação em 14.12.2004 e na forma revisada em 1.3.2005.

2 Eng.-Agr., aluno de Pós-graduação, Dep. de Produção Vegetal-Agricultura, FCA/UNESP, <neumarcio@fca.unesp.br>. ${ }^{3}$ Prof. Livre Docente, Dep. de Produção Vegetal-Agricultura, FCA/UNESP; ${ }^{4}$ Aluno de graduação - FCA/UNESP, Caixa Postal 237, 18603-970 Botucatu-SP. 
P. decumbens species: 2,4-D amina (U-46 D FLUID 720) at 2,880 g a.e. ha ${ }^{-1}$; diquat (REWARD 240) at $480 \mathrm{~g}$ a.i. ha ${ }^{-1}$; propanyl (STAM 480) at 2, $880 \mathrm{~g}$ a.i. ha ; glyphosate (RODEO 480) at 3,360 g a.e. ha $\mathrm{g}^{-1}$ plus the surfactante Aterbane BR $\left(0.5 \% v v^{-1}\right)$; glyphosate + propanyl at

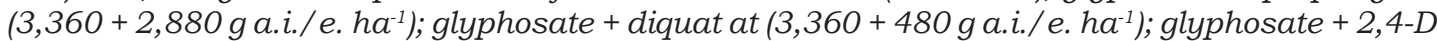
at $\left(3,360+2,880 \mathrm{~g}\right.$ a.e. $\left.h \mathrm{a}^{-1}\right)$; propanyl $+2,4-\mathrm{D}$ at $\left(2,880+2,880 \mathrm{~g}\right.$ a.i./ e. $\left.\mathrm{ha}^{-1}\right)$; diquat + 2, 4-D at $\left(480+2,880 \mathrm{~g}\right.$ a.i./ e. $\left.\mathrm{ha}^{-1}\right)$ and a control without herbicide. The herbicides were applied with the help of a stationary sprayer, pressurized to air compressed and equipped with a reservoir of 2 liters, "Teejet" XR11002VS nozzle tips with a spraying consumption of $200 \mathrm{~L} \mathrm{ha} \mathrm{H}^{-1}$. The weeds were visually evaluated through a scale of percentile of grades (0 to 100\%), and the dry matter of the plants was also evaluated. It was verified that the chemical control determined is a good alternative for the species $\boldsymbol{A}$. philoxeroides, $\boldsymbol{E}$. anagallis and $\boldsymbol{P}$. decumbens. The herbicide mix could increase control efficiency. $\boldsymbol{E}$. anagallis showed high sensitivity to herbicide action. However, the species $\boldsymbol{A}$. philoxeroides and $\boldsymbol{P}$. decumbens showed a high cpacity to regenerate mainly when contact action herbicides were applied.

Key words: 2,4-D, diquat, imazapyr, glyphosate, weed.

\section{INTRODUÇÃO}

As plantas aquáticas podem colonizar os mais diferentes tipos de ambiente, e várias espécies podem suportar longos períodos de estiagem. Isso ocorre pelo fato de as plantas aquáticas apresentarem alta capacidade de adaptação e alta amplitude ecológica (Esteves, 1998). Plantas aquáticas que proliferam em reservatórios, rios e lagos podem provocar vários inconvenientes, como: acúmulo de lixo e sedimentos agricolas, proliferação de vetores de doenças, obstrução de hidrovias, prejuízos ao turismo regional e à pesca, sendo, nesses casos, consideradas daninhas (Martins et al., 1999; Tanaka et al., 2002; Thomaz, 2002).

Príncipe et al. (1997) afirmam que um dos principais transtornos ocasionados pela incidência de plantas aquáticas em grande quantidade, notadamente em épocas de cheias, está relacionado com a redução da potência de cada unidade geradora de energia das usinas hidrelétricas, pois o acúmulo de detritos vegetais nas grades da tomada de água de uma unidade geradora impede que esta gere sua potência máxima, reduzindo assim a capacidade de produção de energia da usina. Os mesmos autores relatam que no período de 1990 a 1997 foram substituídas 757 unidades de grades de tomada de água da usina hidrelétrica de Jupiá (Estado de São Paulo), que apresentavam rompidas ou deformadas.

Em levantamento realizado no reservatório operado pela AES Tietê S.A. no município de Barra Bonita/SP, foram identificadas 17 espécies de plantas aquáticas distribuídas em 11 famílias; dentre as espécies mais freqüentes, destacam-se Alternanthera philoxeroides (Mart.) Griseb. e Enhydra anagallis Gardner (Carvalho et al., 2003).

A. philoxeroides é originária da América do Sul, pertencente à família Amaranthaceae, podendo ser encontrada desde o oeste dos Estados Unidos até a Argentina; ocorre também na África, na Índia, no sudeste da Ásia e na Austrália. É uma planta aquática emersa enraizada, infestante de várzeas úmidas e áreas alagadas, inicialmente a partir das margens, podendo parte do vegetal desprender-se e flutuar (Jain, 1975; Kissmann \& Groth, 1997, 1999; Gunasekera \& Bonila, 2001).

E. anagallis é pertencente à família Compositae e ocorre na Região Sul do Brasil, no Uruguai, no Paraguai e no nordeste da Argentina; na América do Sul ocorrem cerca de 10 espécies. É uma planta perene, infestante de áreas inundadas, podendo formar densa cobertura sobre a superficie da água.

A espécie Pycreus decumbens é infestante altamente agressiva em área com baixa lâmina de água, sendo mais comum a presença em reservatórios de pequeno porte, como represas e açudes. Pertencente à família Cyperaceae, é uma planta perene nativa do Brasil comum nos Estados de São Paulo e Paraná (Kissmann \& Groth, 1997,1999). 
Essas espécies podem invadir represas rurais e diminuir a vida útil do reservatório, facilitando o acúmulo de material erodido e reduzindo a qualidade e a capacidade de armazenamento de água, bem como aumentar as perdas por transpiração (Pitelli, 1998).

Dentre as alternativas de manejo de plantas daninhas aquáticas, destaca-se o uso de herbicidas. Segundo Haller (1998), para o controle químico de plantas aquáticas nos Estados Unidos existem apenas seis herbicidas registrados: 2,4-D, diquat, cobre, endothall, fluridone e glyphosate. Contudo, no Brasil existem poucos estudos referentes ao controle químico dessas espécies, havendo, portanto, a necessidade de intensificar esforços nessa área, a fim de diminuir ou evitar os prejuízos causados por essas espécies em reservatórios de hidrelétricas e outros, além de identificar herbicidas eficientes no controle e seguros para os ambientes aquáticos.

Os herbicidas propanil e imazapyr destacam-se como alternativa, por demonstrarem elevado potencial para uso em ambientes aquáticos no controle de plantas daninhas (Kay, 1995; Martins et al., 1999; Carbonari et al., 2003, 2004). Vários estudos realizados por diferentes pesquisadores têm comprovado a eficiência do controle químico e a sua segurança sobre os organismos aquáticos através do uso de diversos herbicidas (Schultz \& Gangstad, 1976; Langeland \& Warner, 1986; Carpentier et al., 1988; Murphy \& Barrett, 1990; Hofstra et al., 2001; Martins et al., 1999, 2002; Neves et al., 2002; Parsons et al., 2001).

As espécies do presente estudo possuem poucas informações sobre morfologia e biologia, bem como estudos de controle químico que possam auxiliar nas tomadas de decisão de manejo. Assim, o objetivo deste trabalho foi avaliar a eficiência de diferentes herbicidas no controle químico de plantas de A. philoxeroides, E. anagallis e $P$. decumbens em caixa d'água.

\section{MATERIAL E MÉTODOS}

O experimento foi instalado e conduzido no Núcleo de Pesquisas Avançadas em Matologia (NUPAM), pertencente ao Departamento de Produção Vegetal da Faculdade de Ciências Agronômicas/UNESP, campus de Botucatu-SP.
As plantas de A. philoxeroides, E. anagallis e P. decumbens foram coletadas em 27.06.2004 na várzea da Fazenda Edgardia, pertencente à Faculdade de Ciências Agronômicas/UNESP, campus de Botucatu-SP. Devido à alta capacidade de reprodução por meios vegetativos, foram retiradas mudas, as quais foram transplantadas para as caixas no mesmo dia da coleta. As plantas foram cultivadas em caixas plásticas de cor preta, com dimensões da $15 \mathrm{x}$ $15 \times 11 \mathrm{~cm}$, contendo uma camada de $6 \mathrm{~cm}$ de solo como substrato.

Para A. philoxeroides foram colocadas duas mudas por vaso, enquanto para as espécies $E$. anagallis e $P$. decumbens colocaram-se três mudas por vaso. Foi mantida uma lâmina de água de $5 \mathrm{~cm}$ durante todo o período experimental. As características físicas do solo utilizado eram: 749, 52 e $199 \mathrm{~g} \mathrm{~kg}^{-1}$ de areia, silte e argila, respectivamente. Já os resultados da análise química evidenciaram $\mathrm{pH}$ em $\mathrm{CaCl}_{2}$ de 4,5; teor de matéria orgânica de 26,4 $\mathrm{g} \mathrm{dm}^{-3}$; CTC de 53,0 e P de 4,3 $\mathrm{mg} \mathrm{dm}^{-3}$; valores de $0,1,12,7$ e $1,5 \mathrm{mmol}_{\mathrm{c}} \mathrm{dm}^{-3}$ para $\mathrm{K}$, $\mathrm{Ca}$ e $\mathrm{Mg}$, respectivamente; e saturação por bases de $27 \%$.

Quando as plantas atingiram o seu pleno desenvolvimento (antes do florescimento), foram aplicados os seguintes herbicidas para as espécies A. philoxeroides e E. anagallis, aos 43 e 66 dias após o transplantio, respectivamente: 2,4-D amina (U-46 D FLUID 720) a 2.880 g e.a. ha ${ }^{-1}$; diquat (REWARD 240) a 480 g i.a. ha ${ }^{-1}$; imazapyr (ARSENAL 250) a 500 e 750 g e.a. ha ${ }^{-1}$; glyphosate (RODEO 480) a $3.360 \mathrm{~g}$ e.a. ha $\mathrm{ha}^{-1} \mathrm{com}$ e sem o surfatante Aterbane BR $\left(0,5 \% \mathrm{v} \mathrm{v}^{-1}\right)$; glyphosate + diquat $(3.360+480$ g i./e.a. ha-1); glyphosate $+2,4-\mathrm{D}$ $\left(3.360+2.880\right.$ g e.a. ha $\left.{ }^{-1}\right)$; e diquat $+2,4-\mathrm{D}$ $\left(480+2.880 \mathrm{~g}\right.$ i./e.a. ha $\left.{ }^{-1}\right)$; além de uma testemunha sem aplicação de herbicida. Para $P$. decumbens foram aplicados os seguintes herbicidas, aos 76 dias após o transplantio: 2,4-D amina (U-46 D FLUID 720) a 2.880 g e.a. ha ${ }^{-1}$; diquat (REWARD 240) a 480 g i.a. ha ${ }^{-1}$; propanil (STAM 480) a 2.880 g i.a. ha ${ }^{-1}$; glyphosate (RODEO 480) a 3.360 g e.a. ha ${ }^{-1}$ mais o surfatante Aterbane BR $\left(0,5 \% \mathrm{v} \mathrm{v}^{-1}\right)$; glyphosate + propanil $\left(3.360+2.880\right.$ g i./e.a. ha $\left.{ }^{-1}\right)$; glyphosate + diquat $(3.360+480$ g i./e.a. ha-1); glyphosate $+2,4-\mathrm{D}(3.360+2.880$ g e.a. ha-1 $)$; propanil + 2,4-D $\left(2.880+2.880\right.$ g i. /e.a. ha $\left.{ }^{-1}\right) ;$ e

Planta Daninha, Viçosa-MG, v. 23, n. 2, p. 335-342, 2005 
diquat +2 ,4-D $\left(480+2.880\right.$ g i./e.a. ha $\left.{ }^{-1}\right)$, além de uma testemunha sem aplicação de herbicida.

Os herbicidas foram aplicados com um pulverizador estacionário, pressurizado a ar comprimido e equipado com um reservatório de 2 litros. Utilizou-se um consumo de calda de 2001 ha $^{-1}$. A barra de aplicação estava equipada com quatro pontas de jato plano, Teejet XR11002VS, distanciados $50 \mathrm{~cm}$ entre si.

As condições de temperatura e umidade relativa do ar do laboratório no momento da aplicação dos herbicidas estão apresentadas na Tabela 1.

As avaliações de controle das plantas daninhas foram feitas de forma visual, através de uma escala de percentual de notas, em que 0 (zero) corresponde a nenhuma injúria demonstrada pela planta e 100 (cem) à morte das plantas (SBCPD, 1995). As avaliações de controle para as espécies $A$. philoxeroides e E. anagallis foram feitas aos 3, 7, 14, 21, $28 \mathrm{e}$ 49 dias após aplicação dos herbicidas. Já para a espécie $P$. decumbens as avaliações de controle foram realizadas aos 3, 7, 14, 21, 28 e 42 dias após aplicação dos herbicidas. Os parâmetros utilizados para o estabelecimento das notas foram: inibição do crescimento, quantidade e uniformidade das injúrias, capacidade de rebrota das plantas, quantidade de plantas mortas e acúmulo de biomassa. Ao final do experimento foi avaliado o acúmulo de massa seca das plantas.

Os tratamentos foram dispostos em um delineamento experimental inteiramente casualizado com quatro repetições, em casa de vegetação. Os resultados foram submetidos à análise de variância pelo teste $\mathrm{F}$, e as médias dos tratamentos, comparadas pelo teste $t$ $(p>0,05)$.

\section{RESULTADOS E DISCUSSÃO}

Na Tabela 2 estão apresentados os resultados da porcentagem de controle de plantas de A. philoxeroides em diferentes períodos de avaliação, após a aplicação de herbicidas, e os valores de massa seca da parte aérea e das raízes.

Aos três dias após a aplicação dos herbicidas (DAA), observou-se que apenas os tratamentos com imazapyr e glyphosate com e sem adjuvante não apresentaram sintomas visuais de toxicidade, devido, talvez, ao modo de ação desses herbicidas sistêmicos, que necessitam de um período maior de tempo para proporcionar injúrias às plantas. A partir dos sete dias, todos os herbicidas já proporcionavam alguma injúria às plantas, e aos 21 DAA os tratamentos com imazapyr e o glyphosate sem adjuvante evidenciaram controle superior a $98,0 \%$; as plantas iniciaram uma rebrota pouco vigorosa aos 28 DAA.

O aparecimento de injúrias proporcionado pelo herbicida diquat é rápido na presença de luz, pois ocorre no cloroplasto a redução do íon bipiridílio a radical bipiridílio, liberando no processo água oxigenada e promovendo, dessa forma, queimaduras severas nas folhas, evoluindo posteriormente para necrose (Rodrigues \& Almeida, 1998; Nelson \& Getsinger, 2000; Martins et al., 2002; Neves et al., 2002). Os tratamentos com diquat e glyphosate + diquat demonstraram controle acima de $90,0 \%$ a partir dos 7 DAA. Entretanto, nesse período ocorreu abscisão do terço superior do caule das plantas, o que pode ter prejudicado a translocação dos herbicidas, favorecendo assim a rebrota da planta a partir do terço inferior do caule aos 14 DAA e reduzindo o controle a zero aos 21 DAA em ambos os tratamentos. O 2,4-D, aplicado de forma isolada e em mistura com o

Tabela 1 - Horário da aplicação, umidade relativa e temperatura do ar durante a pulverização dos herbicidas nas espécies avaliadas. Botucatu-SP, 2004

\begin{tabular}{|l|c|c|c|c|c|c|}
\hline \multirow{2}{*}{ Condição } & \multicolumn{2}{|c|}{ Alternanthera philoxeroides } & \multicolumn{2}{|c|}{ Enhydra anagallis } & \multicolumn{2}{c|}{ Pycreus decumbens } \\
\cline { 2 - 7 } & \multicolumn{2}{|c|}{$09 / 08 / 04$} & \multicolumn{2}{|c|}{$01 / 09 / 04$} & \multicolumn{2}{c|}{$11 / 09 / 04$} \\
\cline { 2 - 7 } & Inicial & Final & Inicial & Final & Inicial & Final \\
\hline Horário da aplicação & $9 \mathrm{~h} 00$ & $9 \mathrm{~h} 45$ & $9 \mathrm{~h} 50$ & $10 \mathrm{~h} 30$ & $9 \mathrm{~h} 20$ & $10 \mathrm{~h} 00$ \\
\hline Umidade relativa do ar (\%) & 60 & 62 & 50 & 52 & 51 & 50 \\
\hline Temperatura do ar $\left({ }^{\circ} \mathrm{C}\right)$ & 20,2 & 20,3 & 23,5 & 23,6 & 24,7 & 24,5 \\
\hline
\end{tabular}


Tabela 2 - Porcentagem de controle de Alternanthera philoxeroides em diferentes períodos de avaliação após a aplicação de herbicidas e acúmulo da massa seca na parte aérea e nas raízes. Botucatu-SP, 2004

\begin{tabular}{|c|c|c|c|c|c|c|c|c|c|}
\hline \multirow{2}{*}{ Tratamento } & \multirow{2}{*}{$\frac{\text { Dose }}{\left.\text { (g i./e.a. } \text { ha }^{-1}\right)}$} & \multicolumn{6}{|c|}{ Dias após a aplicação } & \multicolumn{2}{|c|}{ Massa seca $(\mathrm{g})$} \\
\hline & & 3 & 7 & 14 & 21 & 28 & 49 & Parte aérea & Raiz \\
\hline 1. Testemunha & - & - & - & - & - & - & - & $2,060 \mathrm{a}$ & $1,275 \mathrm{a}$ \\
\hline 2. 2,4-D & 2.880 & $10,5 \mathrm{~d}$ & $43,8 \mathrm{c}$ & $88,8 \mathrm{e}$ & $95,8 \mathrm{c}$ & $95,3 \mathrm{bc}$ & $71,3 \mathrm{c}$ & $1,185 \mathrm{c}$ & $0,695 \mathrm{~b}$ \\
\hline 3. diquat & 480 & $58,8 \mathrm{~b}$ & $95,8 \mathrm{a}$ & $95,8 \mathrm{bc}$ & $0,0 \mathrm{~d}$ & $0,0 \mathrm{~d}$ & $0,0 \mathrm{~d}$ & $1,463 \mathrm{bc}$ & $0,460 \mathrm{~b}$ \\
\hline 4. imazapyr & 500 & $0,0 \mathrm{e}$ & $28,8 \mathrm{de}$ & $94,5 \mathrm{c}$ & $98,3 \mathrm{~b}$ & $93,8 \mathrm{c}$ & $0,0 \mathrm{~d}$ & $1,308 \mathrm{~b}$ & $0,603 \mathrm{~b}$ \\
\hline 5. imazapyr & 750 & $0,0 \mathrm{e}$ & 26,3 e & $91,3 \mathrm{de}$ & $99,0 \mathrm{ab}$ & $96,8 \mathrm{abc}$ & $0,0 \mathrm{~d}$ & $1,270 \mathrm{~b}$ & $0,585 \mathrm{~b}$ \\
\hline 6. glyphosate & 3.360 & $0,0 \mathrm{e}$ & 33,8 cde & $08,5 \mathrm{ab}$ & $99,3 \mathrm{ab}$ & $95,0 \mathrm{bc}$ & $75,0 \mathrm{bc}$ & $0,390 \mathrm{~d}$ & $0,143 \mathrm{c}$ \\
\hline 7. glyphosate+Aterbane BR $\left(0,5 \% \mathrm{v} \mathrm{v}^{-1}\right)$ & 3.360 & $0,0 \mathrm{e}$ & $40,0 \mathrm{~cd}$ & $97,8 \mathrm{ab}$ & $100,0 \mathrm{a}$ & $100,0 \mathrm{a}$ & $100,0 \mathrm{a}$ & $0,0 \mathrm{~d}$ & $0,0 \mathrm{c}$ \\
\hline 8. glyphosate + diquat & $3.360+480$ & $72,5 \mathrm{a}$ & $94,5 \mathrm{a}$ & $93,8 \mathrm{a}$ & $0,0 \mathrm{~d}$ & $0,0 \mathrm{~d}$ & $0,0 \mathrm{~d}$ & $1,925 \mathrm{ab}$ & $0,620 \mathrm{~b}$ \\
\hline 9. glyphosate $+2,4-\mathrm{D}$ & $3.360+2.880$ & $18,8 \mathrm{c}$ & $80,0 \mathrm{~b}$ & $98,5 \mathrm{ab}$ & $99,0 \mathrm{ab}$ & $99,0 \mathrm{ab}$ & $91,3 \mathrm{ab}$ & $1,473 \mathrm{bc}$ & $0,488 \mathrm{~b}$ \\
\hline 10. diquat $+2,4-\mathrm{D}$ & $480+2.880$ & $60,0 \mathrm{~b}$ & $95,8 \mathrm{a}$ & $98,8 \mathrm{a}$ & $99,0 \mathrm{ab}$ & $99,0 \mathrm{ab}$ & $91,3 \mathrm{ab}$ & $1,363 \mathrm{~b}$ & $0,658 \mathrm{~b}$ \\
\hline $\mathrm{F}_{\text {testemunha }}$ & & $141,428 * *$ & $73,290 * *$ & $1.007,123 * *$ & $11.233,089 * *$ & $968,670 * *$ & $62,363^{* *}$ & $11,968 * *$ & $11,413^{* *}$ \\
\hline $\mathrm{CV}(\%)$ & & 22,6 & 15,1 & 2,2 & 1,3 & 4,4 & 27,1 & 29,1 & 36,6 \\
\hline d.m.s. & & '"7,199 & 11,707 & 2,759 & 1,298 & 4,350 & 16,792 & 0,523 & 0,292 \\
\hline
\end{tabular}

Médias seguidas de mesma letra, na coluna, não diferem estatisticamente entre si pelo teste $\mathrm{t}(\mathrm{p}>0,05)$.

** valor significativo pelo test $\mathrm{F}(\mathrm{p}>0,01)$.

glyphosate e o diquat, apresentou eficiência de controle da ordem de 95,8, 99,0 e 99,0\%, respectivamente, aos 21 DAA. No entanto, as plantas apresentaram rebrotas através das gemas do caule que estavam imersos na água, aos 28 DAA. Esses resultados confirmam os obtidos por Gangstad et al. (1975).

Dentre os herbicidas avaliados, o glyphosate sem e com adjuvante apresentou média de redução da massa seca da parte aérea superior a $80 \%$; para as raízes, os valores foram da ordem de $100 \%$, aos 49 DAA. Em todos os tratamentos ocorreram maiores reduções da massa seca das raízes em relação à da parte aérea.

$\mathrm{Na}$ Tabela 3 estão apresentados os resultados de porcentagem de controle de E. anagallis em diferentes períodos de avaliação após a aplicação de herbicidas e os valores da massa seca da parte aérea e da raiz. Pode-se observar que apenas os tratamentos com imazapyr não mostraram sintomas visuais de controle aos 3 DAA, obtendo, portanto, controle satisfatório de $98,5 \%$, independentemente da dose aplicada aos 49 DAA, e com reduções no acúmulo de massa seca da parte aérea e das raízes na ordem de 80,2 $62,5 \%$ e $91,2-81,3 \%$, respectivamente para as doses de 500 e $750 \mathrm{~g}$ e.a. ha ${ }^{-1}$. Os herbicidas 2,4-D e diquat e a mistura de glyphosate + 2,4-D apresentaram eficiência de $100 \%$ a partir dos 7 DAA, e a mistura de diquat + 2,4-D, somente a partir dos 14 DAA.

Os tratamentos com glyphosate sem e com a adição do adjuvante Aterbane evidenciaram controle superior a $90 \%$ aos 14 DAA, porém as plantas iniciaram rebrotas aos 21 DAA. Da mesma forma, as plantas tratadas com a mistura de glyphosate + diquat rebrotaram a partir dos 14 DAA. Essa ineficiência no controle pode ter ocorrido, talvez, devido à condição de crescimento das plantas; no momento da aplicação, partes imersas do vegetal ou protegidas por ramos maiores podem não ter recebido o produto. Outra hipótese pode estar relacionada a um possivel efeito antagônico entre os herbicidas glyphosate e diquat no controle da espécie E. anagallis. Diversos pesquisadores, a exemplo de Martins et al. (1999, 2002), Neves et al. (2002) e Carbonari et al. (2004), também encontraram dificuldades no controle químico das plantas daninhas aquáticas Eicchornia crassipes, Pistia stratiotes, Panicum repens, Paspalum repens e Typha subulata no que se refere à rebrota das plantas após alguns dias da aplicação dos herbicidas.

Podem-se verificar, na Tabela 4 , os resultados de porcentagem de controle de $P$. decumbens em diferentes periodos de avaliação após a aplicação de herbicidas e os valores da massa seca da parte aérea e das raízes. As parcelas que receberam diquat, 
propanil e as misturas de glyphosate + diquat e diquat $+2,4-\mathrm{D}$ apresentaram evidências de rebrota aos 21 DAA e porcentagem de controle inferior a $70 \%$.

As misturas de propanil + 2,4-D e glyphosate + propanil foram ineficientes no controle de $P$. decumbens, permitindo rebrotas das plantas a partir dos 28 e 42 DAA, respectivamente. Contudo, a mistura propanil + 2,4-D promoveu a redução da massa seca da parte aérea em cerca de $93,4 \%$ e das raízes em $87,9 \%$.
O glyphosate mais o adjuvante Aterbane apresentou eficiência inferior a $35 \%$ aos 21 DAA, chegando a zero aos 42 DAA. Entretanto, o 2,4-D e a mistura de glyphosate $+2,4-\mathrm{D}$ mostraram eficiência de 97,3 e $100 \%$ aos 42 DAA, respectivamente, evidenciando um possivel sinergismo entre os dois herbicidas. Parsons et al. (2001) observaram que plantas de Myriophyllum spicatum tratadas com 2,4-D no lago Loon, em Washington (EUA), apresentaram redução da biomassa em torno de $98 \%$ aos 42 DAA; após um ano da aplicação

Tabela 3 - Porcentagem de controle de Enhydra anagallis em diferentes períodos de avaliação após a aplicação de herbicidas e acúmulo da massa seca na parte aérea e nas raízes. Botucatu-SP, 2004

\begin{tabular}{|c|c|c|c|c|c|c|c|c|c|}
\hline \multirow{2}{*}{ Tratamento } & \multirow{2}{*}{$\frac{\text { Dose }}{\left.\text { (g i./e.a. } \text { ha }^{-1}\right)}$} & \multicolumn{6}{|c|}{ Dias após a aplicação } & \multicolumn{2}{|c|}{ Massa seca (g) } \\
\hline & & 3 & 7 & 14 & 21 & 28 & 49 & Parte aérea & Raiz \\
\hline 1. Testemunha & - & - & - & - & - & - & - & $9,000 \mathrm{a}$ & $2,620 \mathrm{a}$ \\
\hline 2. $2,4-\mathrm{D}$ & 2.880 & 13,0 de & $100,0 \mathrm{a}$ & $100,0 \mathrm{a}$ & $100,0 \mathrm{a}$ & $100,0 \mathrm{a}$ & $100,0 \mathrm{a}$ & $0,000 \mathrm{~d}$ & $0,000 \mathrm{~b}$ \\
\hline 3. diquat & 480 & $62,5 \mathrm{a}$ & $100,0 \mathrm{a}$ & $100,0 \mathrm{a}$ & $100,0 \mathrm{a}$ & $100,0 \mathrm{a}$ & $100,0 \mathrm{a}$ & $0,000 \mathrm{~d}$ & $0,000 \mathrm{~b}$ \\
\hline 4. imazapyr & 500 & $0,0 \mathrm{f}$ & $10,8 \mathrm{c}$ & $20,0 \mathrm{~b}$ & $40,0 \mathrm{~d}$ & $60,0 \mathrm{bc}$ & $98,5 \mathrm{a}$ & $1,778 \mathrm{bc}$ & $0,983 \mathrm{~b}$ \\
\hline 5. imazapyr & 750 & $0,0 \mathrm{f}$ & $11,3 \mathrm{c}$ & $36,3 \mathrm{~b}$ & $74,3 \mathrm{bc}$ & $71,3 \mathrm{abc}$ & $98,5 \mathrm{a}$ & $0,788 \mathrm{bcd}$ & $0,490 \mathrm{~b}$ \\
\hline 6. glyphosate & 3.360 & $15,0 \mathrm{~d}$ & $75,0 \mathrm{~b}$ & $92,3 \mathrm{a}$ & $92,5 \mathrm{ab}$ & $90,0 \mathrm{ab}$ & $75,0 \mathrm{ab}$ & $0,808 \mathrm{bcd}$ & $0,250 \mathrm{~b}$ \\
\hline 7. glyphosate+Aterbane BR $\left(0,5 \% \mathrm{~V} \mathrm{v}^{-1}\right)$ & 3.360 & $10,0 \mathrm{e}$ & $76,3 \mathrm{~b}$ & $90,0 \mathrm{a}$ & $89,5 \mathrm{abc}$ & $85,8 \mathrm{ab}$ & $25,0 \mathrm{~cd}$ & $0,478 \mathrm{~cd}$ & $0,128 \mathrm{~b}$ \\
\hline 8. glyphosate + diquat & $3.360+480$ & $49,3 \mathrm{~b}$ & $97,5 \mathrm{a}$ & $96,3 \mathrm{a}$ & $72,5 \mathrm{c}$ & $50,0 \mathrm{c}$ & $50,0 \mathrm{bc}$ & $2,075 \mathrm{~b}$ & $0,863 \mathrm{~b}$ \\
\hline 9. glyphosate $+2,4-\mathrm{D}$ & $3.360+2.880$ & $21,3 \mathrm{c}$ & $100,0 \mathrm{a}$ & $100,0 \mathrm{a}$ & $100,0 \mathrm{a}$ & $100,0 \mathrm{a}$ & $100,0 \mathrm{a}$ & $0,000 \mathrm{~d}$ & $0,000 \mathrm{~b}$ \\
\hline 10. diquat + 2,4-D & $480+2.880$ & $50,5 \mathrm{~b}$ & $99,5 \mathrm{a}$ & $100,0 \mathrm{a}$ & $100,0 \mathrm{a}$ & $100,0 \mathrm{a}$ & $100,0 \mathrm{a}$ & $0,000 \mathrm{~d}$ & $0,000 \mathrm{~b}$ \\
\hline $\mathrm{F}_{\text {testemunha }}$ & & $463,736^{* *}$ & $629,722 * *$ & $41,711 * *$ & $26,132 * *$ & $9,288 * *$ & $6,584 * *$ & $25,065^{* *}$ & $3,934 * *$ \\
\hline $\mathrm{CV}(\%)$ & & 9,8 & 5,0 & 16,4 & 16,8 & 27,9 & 38,7 & 73,4 & 155,0 \\
\hline d.m.s. & & 3,136 & 4,878 & 17,398 & 18,680 & 30,504 & 41,703 & 1,582 & 1,194 \\
\hline
\end{tabular}

Médias seguidas de mesma letra, na coluna, não diferem estatisticamente entre si pelo teste $t(p>0,05)$.

** valor significativo pelo test $\mathrm{F}(\mathrm{p}>0,01)$.

Tabela 4 - Porcentagem de controle de Pycreus decumbens em diferentes períodos de avaliação após a aplicação de herbicidas e acúmulo da massa seca na parte aérea e nas raízes. Botucatu-SP, 2004

\begin{tabular}{|c|c|c|c|c|c|c|c|c|c|}
\hline \multirow{2}{*}{ Tratamento } & \multirow{2}{*}{$\frac{\text { Dose }}{\left(\text { g i./e.a. } \text { ha }^{-1}\right)}$} & \multicolumn{6}{|c|}{ Dias após a aplicação } & \multicolumn{2}{|c|}{ Massa seca (g) } \\
\hline & & 3 & 7 & 14 & 21 & 28 & 49 & Parte aérea & Raiz \\
\hline 1. Testemunha & - & - & - & - & - & - & - & $4,838 \mathrm{a}$ & $3,135 \mathrm{a}$ \\
\hline 2. 2,4-D & 2.880 & $4,0 \mathrm{~cd}$ & $78,8 \mathrm{a}$ & $91,3 \mathrm{a}$ & $93,8 \mathrm{a}$ & $95,0 \mathrm{a}$ & $97,3 \mathrm{a}$ & $0,808 \mathrm{~d}$ & 0,490 de \\
\hline 3. diquat & '"' 480 & $15,0 \mathrm{a}$ & $52,5 \mathrm{~b}$ & $51,3 \mathrm{c}$ & $41,3 \mathrm{~cd}$ & $28,8 \mathrm{e}$ & $0,0 \mathrm{~b}$ & $3,085 \mathrm{ab}$ & $2,625 \mathrm{ab}$ \\
\hline 4. imazapyr & 500 & 2,0 de & $12,5 \mathrm{~d}$ & $57,5 \mathrm{c}$ & $41,3 \mathrm{~cd}$ & $37,5 \mathrm{e}$ & $0,0 \mathrm{~b}$ & $2,495 \mathrm{bc}$ & $1,518 \mathrm{bcd}$ \\
\hline 5. imazapyr & 750 & $3,0 \mathrm{~d}$ & $21,3 \mathrm{~d}$ & $33,8 \mathrm{~d}$ & $35,0 \mathrm{~d}$ & $33,8 \mathrm{e}$ & $0,0 \mathrm{~b}$ & $2,483 \mathrm{bc}$ & 1,150 cde \\
\hline 6. glyphosate & 3.360 & "'2,0 de & $16,3 \mathrm{~d}$ & $58,8 \mathrm{c}$ & $66,3 \mathrm{~b}$ & $72,5 \mathrm{bc}$ & $21,3 \mathrm{~b}$ & $1,508 \mathrm{~cd}$ & 1,205 bcde \\
\hline 7. glyphosate+Aterbane $\mathrm{BR}\left(0,5 \% \mathrm{v} \mathrm{v}^{-1}\right)$ & 3.360 & "'8,8 b & $33,8 \mathrm{c}$ & $60,0 \mathrm{c}$ & $47,5 \mathrm{c}$ & $45,0 \mathrm{de}$ & $0,0 \mathrm{~b}$ & $3,560 \mathrm{ab}$ & 1,945 bc \\
\hline 8. glyphosate + diquat & $3.360+480$ & $8,8 \mathrm{~b}$ & $87,5 \mathrm{a}$ & $96,0 \mathrm{a}$ & $95,8 \mathrm{a}$ & $96,0 \mathrm{a}$ & $100,0 \mathrm{a}$ & $0,0 \mathrm{~d}$ & $0,0 \mathrm{e}$ \\
\hline 9. glyphosate $+2,4-\mathrm{D}$ & $3.360+2.880$ & $12,5 \mathrm{a}$ & $82,5 \mathrm{a}$ & $98,5 \mathrm{a}$ & $97,0 \mathrm{a}$ & $84,8 \mathrm{ab}$ & $75,0 \mathrm{a}$ & $0,320 \mathrm{~d}$ & $0,380 \mathrm{de}$ \\
\hline 10. diquat + 2,4-D & $480+2.880$ & '" $6,3 \mathrm{bc}$ & $53,8 \mathrm{~b}$ & "'m3,8 b' & $68,8 \mathrm{~b}$ & $31,3 \mathrm{~cd}$ & $0,0 \mathrm{~b}$ & $2,430 \mathrm{bc}$ & "1,233 bcde \\
\hline $\mathrm{F}_{\text {testemunha }}$ & & $31,304 * *$ & $65,592 * *$ & $75,165^{* *}$ & $54,612 * *$ & $26,771 * *$ & $17,368 * *$ & $9,032 * *$ & $4,535^{* *}$ \\
\hline $\mathrm{CV}(\%)$ & & 28,3 & 17,9 & 11,3 & 14,6 & 22,1 & 70,9 & 47,2 & 71,3 \\
\hline d.m.s. & & 2,546 & 11,321 & 10,101 & 12,337 & 17,732 & 30,049 & 1,517 & 1,448 \\
\hline
\end{tabular}

Médias seguidas de mesma letra, na coluna, não diferem estatisticamente entre si pelo teste t $(p>0,05)$.

** valor significativo pelo test $\mathrm{F}(\mathrm{p}>0,01)$. 
a redução da biomassa era de $87 \%$. Os herbicidas 2,4-D e glyphosate promoveram controle completo da planta aquática Colocasia esculenta nas doses de 4.300 e 4.500 g e.a. ha-1 , respec- $^{-}$ tivamente, aos 28 DAA. Já o herbicida diquat na dose de 4.200 g e.a. ha ${ }^{-1}$ foi ineficiente, permitindo a rebrota da planta a partir dos 21 DAA (Nelson \& Getsinger, 2000).

Com base nos resultados obtidos, verificase que o controle químico apresenta-se como uma excelente alternativa de manejo para as espécies A. philoxeroides, E. anagallis e $P$. decumbens; a mistura de herbicidas pode aumentar a eficiência no controle e evitar o surgimento de resistência a determinada molécula. E. anagallis mostrou alta suscetibilidade aos herbicidas aplicados. Entretanto, as espécies A. philoxeroides e $P$. decumbens evidenciaram alta capacidade de regeneração, principalmente quando se utilizaram herbicidas de ação de contato.

No entanto, apesar do grande potencial hídrico que o Brasil apresenta e dos prejuízos que essas espécies causam ao uso múltiplo da água, existem poucos estudos realizados no país. Assim, há necessidade de intensificar os estudos sobre a biologia, a morfoanatomia e ecologia dessas espécies, bem como de aumentar as opções de moléculas de herbicidas para uso em ambiente aquático e desenvolver novas técnicas de aplicação de herbicidas, de maneira a melhorar a eficiência e minimizar os efeitos sobre os outros organismos aquáticos; além disso, é preciso determinar o impacto da decomposição do material vegetal no corpo hídrico após ter sido submetido ao controle químico.

\section{LITERATURA CITADA}

CARBONARI, C. A.; MARTINS, D.; TERRA, M. A. Controle de Brachiaria subquadripara e Brachiaria mutica através de diferentes herbicidas aplicados em pósemergência. Planta Daninha. v. 21, p. 79-84, 2003. (Edição especial)

CARBONARI, C. A. et al. Controle químico de Panicum repens e Paspalum repens. Planta Daninha, v. 22, n. 3, p. 453-460, 2004.

CARPENTIER, A. G.; MACKENZIE, D. L.; FRANK, R. Residues and efficacy of two formulations of 2,4-D on aquatic macrophytes in Buckhorn lake, Ontario. J. Aquatic Plant Manag., v. 26, p. 29-37, 1988.
CARVALHO, F. T. et al. Plantas aquáticas e nível de infestação das espécies presentes no reservatório de Barra Bonita, no Tietê. Planta Daninha, v. 21, p. 15-19, 2003. (Edição especial)

ESTEVES, F. A. Fundamentos de limnologia. 2.ed. Rio de Janeiro: Interciência, 1998. 602 p.

GANGSTAD, E. O.; SPENCER, N. R.; FORET, J. A. Towards integrated control of alligatorweed. J. Aquatic Plant Manag., v. 13, p. 30-33, 1975.

GUNASEKERA, L.; BONILA, J. Alligator weed: tasty vegetable in Australian backyards? J. Aquatic Plant Manag., v. 39, p. 17-20, 2001.

HALLER, W. T. Options for mechanical and chemical aquatic weed control. In: CONGRESSO BRASILEIRO DA CIÊNCIA DAS PLANTAS DANINHAS, 21., 1998, Brasília-DF. Resumos... Brasília: 1998. p. 46-53.

HOFSTRA, D. E.; CLAYTON, J. S.; GETSINGER, K. D. Evaluation of selected herbicides for the control of exotic submerged weeds in New Zealand: II. The effects of turbidity on diquat and endothall efficacy. J. Aquatic Plant Manag., v. 39, p. 25-27, 2001.

JAIN, S. C. Aquatic weeds and their management in India. Hyacinth Control J., v. 13, p. 6-8, 1975.

KAY, S. H. Efficacy of wipe-on applications of glyphosate and imazapyr on common reed in aquatic sites. J. Aquatic Plant Manag., v. 33, p. 25-26, 1995.

KISSMANN, K. G.; GROTH, D. Plantas infestantes e nocivas. 2.ed. São Paulo: BASF, 1997. 825 p. Tomo I.

KISSMANN, K. G.; GROTH, D. Plantas infestantes e nocivas. 2.ed. São Paulo. BASF, 1999. 978 p. Tomo II.

LANGELAND, K. A.; WARNER, J. P. Persistence of diquat, endothall and fluridone in ponds. J. Aquatic Plant Manag., v. 24, p. 43-46, 1986.

MARTINS, D. et al. Controle químico de plantas daninhas aquáticas em condições controladas-caixas d'água. Planta Daninha, v. 17, n. 2, p. 289-296, 1999.

MARTINS, D. et al. Controle químico de Pistia stratiotes, Eichhornia crassipes e Salvinia molesta em caixas d'água.

Planta Daninha, v. 20, p. 83-88, 2002. (Edição especial)

MURPHY, K. J.; BARRETT, P. R. F. Chemical control of aquatic weeds. In: PIETERSE, A. H.; MURPHY, K. J. Aquatic weeds: the ecology and management of nuisance aquatic vegetation. Oxford: University Press, 1990. p. $136-173$.

NELSON, L. S.; GETSINGER, K. D. Herbicide evaluation for control of wild taro. J. Aquatic Plant Manag., v. 38, p. 70-72, 2000.

Planta Daninha, Viçosa-MG, v. 23, n. 2, p. 335-342, 2005 
NEVES, T.; FOLONI, L. L.; PITELLI, R. A. Controle químico do aguapé (Eichhornia crassipes). Planta Daninha, v. 20, p. 89-97, 2002. (Edição especial)

PARSONS, J. K.et al. The use 2,4-D for selective control of an early infestation of eurasian watermilfoil in Loon lake, Washington. J. Aquatic Plant Manag., v. 39, p. 117-125, 2001.

PITELLI, R. A. Macrófitas aquáticas no Brasil, na condição de problemáticas. In: WORKSHOP DE CONTROLE DE PLANTAS AQUÁTICAS, 1998, Brasília. Resumos... Brasília: IBAMA, 1998. p. 12-15.

PRINCIPE, C. R.; KURATANI, H.; MELONI, M. L. B. Impactos da afluência de elódeas na operação e manutenção da usina hidroelétrica Eng. Souza Dias (Jupiá)-CESP. In: CONGRESSO BRASILEIRO DA CIÊNCIA DAS PLANTAS DANINHAS, 21., 1997, Caxambu-MG. Resumos... Caxambu: SBCPD, 1997. p. 5-8.
RODRIGUES, B. N.; ALMEIDA, F. S. Guia de herbicidas. 4.ed. Londrina, 1998. 648 p.

SCHULTZ, D. P.; GANGSTAD, E. O. Dissipation of residues of 2,4-D in water, hydrosoil and fish. J. Aquatic Plant Manag., v. 14, p. 43-45, 1976.

SOCIEDADE BRASILEIRA DA CIÊNCIA DAS PLANTAS DANINHAS. Procedimentos para instalação, avaliação e análise de experimentos com herbicidas.

Londrina: 1995. 42 p.

TANAKA, R. H. et al. Ocorrência de plantas aquáticas nos reservatórios da Companhia Energética de São Paulo. Planta Daninha, v. 20, p. 101-111, 2002. (Edição especial)

THOMAZ, S. M. Fatores ecológicos associados à colonização e ao desenvolvimento de macrófitas aquáticas e desafios de manejo. Planta Daninha, v. 20, p. 21-33, 2002. (Edição especial) 